\title{
Gasos Cuínicos
}

\section{Características clínicas y dermatoscópicas del poroma ecrino}

\author{
Claudia Nicklas ${ }^{1}$, Yesenia Valenzuela ${ }^{2}$, Enrique Bellolio ${ }^{3}$
}

\section{RESUMEN}

El poroma ecrino $(\mathrm{PE})$ corresponde a una neoplasia anexial benigna originada de la porción intraepidérmica del conducto de las glándulas sudoríparas. Habitualmente se describe como nódulos eritematosos o color piel ubicados en región palmoplantar, pero sus características clínicas son muy variables.

Se decriben los hallazgos clínicos y dermatoscópicos de una serie de casos de pacientes con PE.

La dermatoscopia puede facilitar significativamente el diagnóstico clínico de la mayoría de los tumores de la piel, sin embargo el PE se caracteriza por un alto grado de variabilidad a la dermatoscopía. Habitualmente se describe un patrón vascular polimorfo con vasos glomerulares, en horquilla, y lineales irregulares con terminaciones semicirculares rodeados de un halo blanco a rosa en forma de caliz o flor de cerezo asociado a áreas rosadas blanquecinas sin estructuras similares a lo observado en melanoma amelanótico, carcinoma espinocelular entre otros tumores benignos y malignos.

El PE puede exhibir características clínicas y dermatoscópicas polimórficas que pueden hacer difícil el diagnóstico y su diferenciación de otras neoplasias cutáneas.

Palabras claves: Poroma ecrino; dermatoscopía; neoplasia anexial cutánea

\section{SUMMARY}

Eccrine poroma $(\mathrm{PE})$ is a benign adnexal neoplasm of the intraepidermal portion of the sweat gland duct. PE typically present as erythematous or flesh-colored nodules on the palms and soles, but the clinical features of PE are highly variable.

Dermatoscopic and clinical findings of patients with EP are described.

Dermoscopy may significantly improves the clinical diagnosis of most skin tumours, but PE is characterised by a high degree of dermoscopic variability. The main dermoscopic features described are polymorphous vascular pattern, whiteto-pink halo surrounding the vessels, pink-white structureless areas, vascular structures of glomerular, hairpin vessels, and linear irregular vessels, mimicking amelanotic melanoma, keratinising tumours and several benign and malignant tumors.

Eccrine poroma may exhibit polymorphic clinical and dermoscopic features making it difficult to diagnosis and differentiate from other skin cancers.

Key words: eccrine poroma; dermoscopy; skin adnexal neoplasms.
$\mathrm{E}$ 1 poroma ecrino es una neoplasia benigna de anexos cutáneos originada a partir de la porción intraepidérmica de los conductos de las glándulas sudoríparas ecrinas o acrosiringio. ${ }^{1}$ Actualmente esta teoría es controvertida, debido a que poromas ubicados en regiones no palmo plantares presentan diferenciación sebácea y pilar sugiriendo un probable doble origen ecrino-apocrino de estos tumores. ${ }^{2}$

${ }^{1}$ Departamento de Dermatología, Facultad de Medicina, Universidad de la Frontera, Temuco, Chile. ${ }^{2}$ Departamento de Dermatología, Facultad de Medicina, Universidad Mayor, Temuco, Chile. ${ }^{3}$ Departamento de Anatomía Patológica. Facultad de Medicina. Universidad de La Frontera, Temuco, Chile

Correspondencia: Claudia Nicklas. Correo electrónico: claudia.nicklas@gmail.com Celular: +56-452325763. Dirección: Manuel Montt \# 112, Temuco, Chile. Código Postal : 4781176.
Los tumores de glándulas sudoríparas constituyen cerca del 1\% de las neoplasias cutáneas primarias. Los poromas comprenden aproximadamente el 10\% de estas lesiones. Suelen afectar a población de entre 40 y 70 años y parecen no tener una predilección por raza o sexo. ${ }^{1}$

Presentamos cinco casos de pacientes con poroma ecrino describiendo los hallazgos clínicos y dermatoscópicos.

\section{REPORTE DE GASOS}

\section{Gaso 1}

Paciente sexo femenino de 80 años, consultó por tumor de $2 \mathrm{~cm}$ en rodilla izquierda de 2 años de evolución, con rápido crecimiento en el último año y episodios de san- 

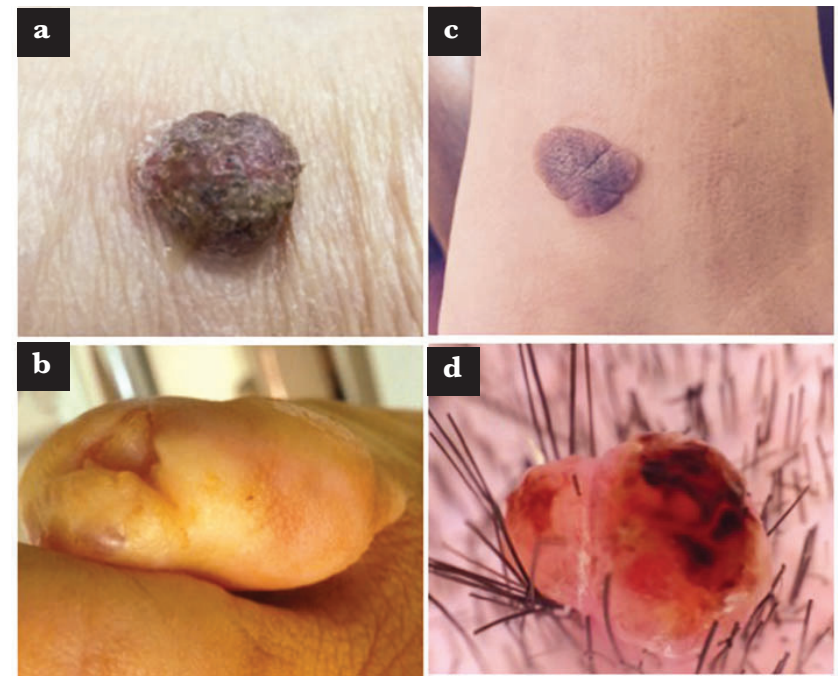

\section{Figura 1}

a. Poroma ecrino rodilla izquierda, tumor eritematoviolaceo exofitico, verrucoso e hiperqueratósico de $2 \mathrm{~cm}$ de diametro simulando un carcinoma espinocelular.

b. PE dorso pie, placa marrón papilomatosa y verrucosa pediculada queratosis seborreica simil.

c. $\mathrm{PE}$ paraungueal.

d. PE cuero cabelludo, a la dermatocopía area eritematosa traslúcida homogenea con lagunas eritematosas rodeadas por halo pálido y escamo costras.

gramiento frente a traumatismo. $\mathrm{Al}$ examen se objetivó un tumor exofítico eritemavioláceo firme, bien delimitado de superficie verrucosa e hiperqueratósica con erosiones y costras serohemáticas en su superficie (Figura 1a). A la dermatocopia destacaba un patrón vascular polimorfo, con vasos glomerulares y lagunas eritematosas rodeadas por halo blanquecino, áreas blanquecino grisaceas sin estructura y escamo costras (Figura 2a).

\section{Gaso 2}

Paciente de sexo femenino de 40 años, consultó por tumor de $2 \mathrm{~cm}$ en dorso pie izquierdo de 3 años de evolución. Al examen destacaba un tumor eritematoviolaceo pediculado de superficie descamativa y verrucosa bien delimitado queratosis seborreica simil (Figura 1b). A la dermatoscopía destacaba la presencia de vasos glomerulares y en horquilla algunos simulando flor de cerezo rodeados de un halo blanquecino asociado a áreas rosadas blanquecinas y gricáseas sin estructura, con cordones blanquecinos interconectados (Figura 2b).
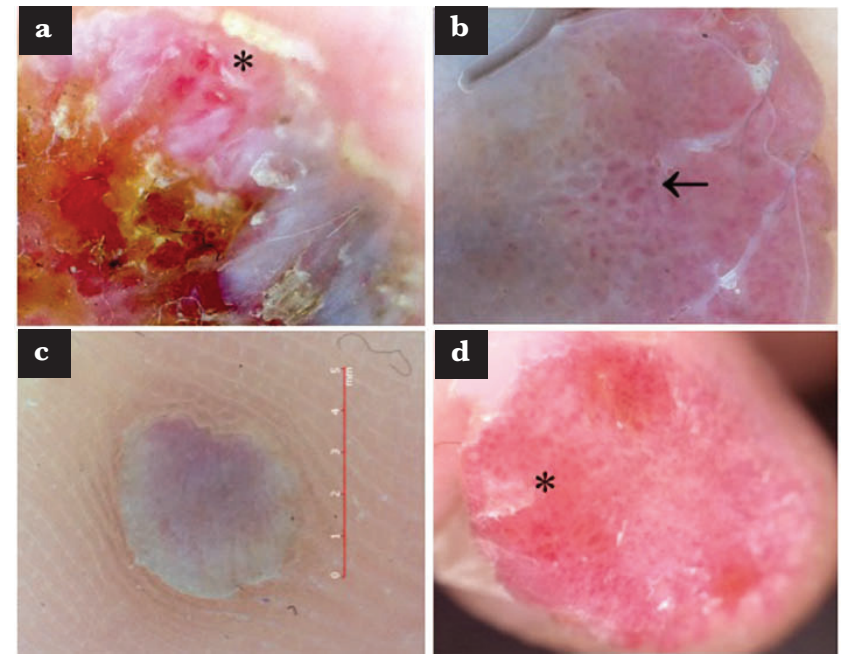

Figura 2

a. Vasos glomerulares y lagunas eritematosas con halo blanquecino (*), areas blanquecina grisaceas sin estructura y escamo costras. b. Vasos simulando flor de cerezo rodeados y cordones blanquecinos interconectados $(\leftarrow)$.

c. Área homogénea rosado violacea rodeado por un área blanquecinas sin estructura en la periferia.

d. Vasos glomerulares rodeados de un halo blanquecino.

\section{Gaso 3}

Paciente de sexo masculino de 73 años, consultó por tumor de $7 \mathrm{~mm}$ plantar derecho de 6 meses de evolución con sensibilidad a la presión profunda. $\mathrm{Al}$ examen presentaba un tumor color piel levemente eritematoso verrucoso bien delimitado. A la dermatoscopía se observaba la presencia un área homogénea sin estructura rosado violacea, opaca, hiperqueratósica con áreas blanquecinas sin estructura en la periferia (Figura 2c).

\section{Gaso 4}

Paciente masculino de 45 años, consultó por tumor de 6 años de evolución de quinto dedo pie derecho. Inicialmente asintomático, pero en los últimos meses sensible al roce del zapato. Al examen presentaba tumor exofitico paraungueal color piel con area eritématoviolacea central (Figura 1c). A la dermatoscopía presentaba en el area central vasos glomerulares rodeados de un halo blanquecino (Figura 2d). 


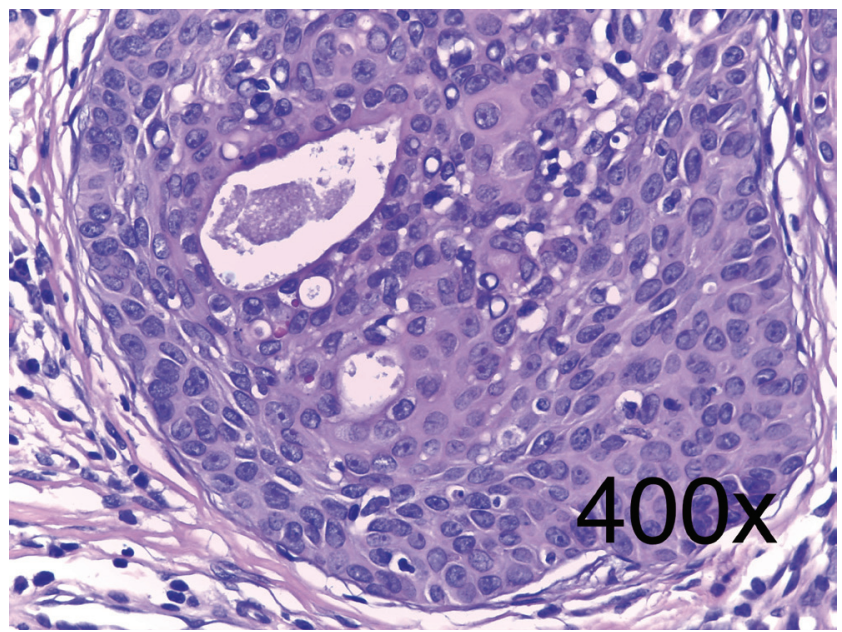

Figura 3

Elongación y proliferación de conductos ecrinos por células epiteliales poroides que conforman nidos y sincio celular con conformación de conductos ecrinos con cutícula eosinófila

\section{Caso 5}

Paciente de sexo masculino de 18 años con cuadro de 3 meses de tumor exofítico en cuero cabelludo con episodios de sangramiento posterior a traumatismos simil a un granuloma telángectásico. $\mathrm{Al}$ examen se observaba un tumor eritematoso costroso serohemático en áreas friables de $5 \mathrm{~mm}$ de diámetro, a la dermatoscopía presentaba área eritematosa traslúcida homogenea con lagunas eritematosas rodeadas por halo pálido y escamo costras (Figura 1d).

En todos los casos se procedió a efectuar biopsia excisional donde a la histopatología se observaban hallazgos similares presentando piel con ortoqueratosis, epidermis con acantosis, destacando elongación y proliferación de conductos ecrinos por células epiteliales poroides que conforman nidos y sincio celular interconectado que se extiendían hasta la dermis reticular profunda. En zonas más profundas se evidenciaba la conformación de conductos ecrinos con cutícula eosinófila distintiva. Las lesiones eran bien delimitadas sin reacción desmoplásica (Figura 3).

\section{Discusión}

La patogénesis del poroma ecrino es desconocida, pero se describen asociaciones con virus papiloma, daño actínico, cicatrices, radiación y trauma. También se ha en- contrado en pacientes con otras enfermedades de la piel como displasia ectodérmica anhidrótica y enfermedad de Bowen. Ocasionalmente, un poroma se puede encontrar como una lesión secundaria dentro de un nevus sebáceo. ${ }^{1,3,4}$

La presentación clínica clásica consiste en una lesión solitaria de aspecto nodular, papular o de placa, de 1 a 2 centímetros de diámetro, de crecimiento lento, de coloración piel, rojizo, rosado y en ocasiones azul o pigmentado, por lo general asintomática. La superficie puede ser lisa, suave, opaca, descamativa, verrucosa, papilomatosa o ulcerada. ${ }^{1,5}$ Esta gran variabilidad en la presentación clínica es la causa de la dificultad en el diagnóstico diferencial con otros tumores cutáneos como el carcinoma espinocelular, carcinoma basocelular o melanoma amelanótico entre otros. ${ }^{1}$

Clásicamente la distribución más frecuente del PE corresponde a superficies acrales (región palmo plantar), 3 a pesar de la creciente evidencia de localizaciones frecuentes en zonas como la cara y cuero cabelludo $(30 \%)$, tronco, extremidades y que solo entre un 20 a $65 \%$ se detectan en región palmoplantar.,

Una presentación rara es el desarrollo de múltiples poromas llegando a más de 100, condición llamada poromatosis, que es más frecuente en pacientes con antecedentes de trastorno linfoproliferativo o exposición a radiación. ${ }^{4}$

La aparición de sintomatología como dolor, ulceración, sangrado espontáneo, prurito repentino y crecimiento rápido se ha relacionado con la tendencia a la malignización en la forma de porocarcinoma. ${ }^{7}$ Se ha reportado que hasta el $18 \%$ de los poromas podrían evolucionar a porocarcinoma en un tiempo promedio de 8.5 años. ${ }^{1,7} \mathrm{El}$ porocarcinoma es un tumor típico de personas mayores de 60 años clinica y dermatoscópicamente muy similar al PE. ${ }^{8}$

En cuanto a los hallazgos a la dermatoscopía predomina un patrón vascular polimorfo con vasos glomerulares, en horquilla y lineales irregulares y ramificados con terminaciones semicirculares en forma de caliz o flor de cerezo rodeados de un halo blanquecino perivascular asociado a áreas rosadas blanquecinas. Siendo estos hallazgos variables y similares a otras neoplasias cutáneas como el patron vascular polimorfo y el halo blanquecino perivascular que simulan un melanoma amelanótico y carcinoma espinocelular respectivamente. Sólo el hallaz- 
go de vasos en un patron de caliz o en flor serían más característicos del PE. ${ }^{1,5,8,9}$ Además se puede observar áreas sin estructura y cordones blanquecinos interconectados, siendo esto último altamente sugerente de poroma. ${ }^{1} \mathrm{En}$ la variante pigmetada se ha descrito la presencia de estructuras azul blanquecinas y globulillares inespecíficas y aperturas tipo comedón. ${ }^{9}$

Histológicamente es un tumor bien delimitado compuesto por una proliferación uniforme de células cúbicas que se extienden desde la epidermis basal a la dermis, asociado a la presencia de estructuras quísticas ductales focales con cutícula eosinófila asociado a un estroma altamente vascularizado. Estas células, que se diferencian claramente de los queratinocitos adyacentes, presentan núcleos monormorfos ovoides, con un patrón no empalizado y discretos nucléolos, y un citoplasma eosinófilo PAS positivo. ${ }^{1}$

El tratamiento es la biopsia excisional para evitar la recurrencia debido al posible potencial maligno y a alteraciones funcionales secundarias a la ubicación palmoplantar. Otras alternativas descritas es el uso de imiquimod crema al 5\%, crioterapia o láser de $\mathrm{CO} 2{ }^{1}{ }^{1}$

El diagnóstico clínico diferencial más importante es con el melanoma amelanótico. Otros diagnósticos incluyen granuloma telangectásico, hemangioma, queratosis seborreica, verrugas, nevos melanocíticos, queratoacantoma, carcinoma espinocelular, carcinoma basocelular y granuloma de la onicocriptosis. ${ }^{1}$

\section{Conclusiones}

El PE debe considerarse como posible diagnóstico frente a una lesión nodular rosada que presenta un patron vascular polimorfo con un halo perivascular blanquecino y áreas sin estructura blanquecino rosadas. Debido a que la presentación clínica del poroma ecrino es variable y poco específica, es importante hacer el diagnóstico diferencial con otras lesiones, principalmente con las de carácter maligno a traves de biopsia excisional.

\section{REFERENCIAS}

1. Sawaya JL, Khachemoune A. Poroma: a review of eccrine, apocrine, and malignant forms. Int J Dermatol 2014;53(9):1053-61

2. Harvell JD, Kerschmann RL, LeBoit PE. Eccrine or apocrine poroma? Six poromas with divergent adnexal differentiation. Am J Dermatopathol 1996;18(1):1-9

3. Johnson RC, Rosenmeier GJ, Keeling JH. A painful step: eccrine poroma. Arch Dermatol 1992;28 (11):1530-1533

4. Deckelbaum S, Touloei K, Shitabata PK, Sire DJ, Horowitz D. Eccrine poromatosis: case report and review of the literature. Int J Dermatol 2014;53(5):543-8

5. Shalom A, Schein O, Landi C, Marghoob A, Carlos B, Scope A. Dermoscopic findings in biopsy-proven poromas. Dermatol Surg 2012;38(7Pt1):1091-6

6. Betti R1, Bombonato C, Cerri A, Moneghini L, Menni S. Unusual sites for poromas are not very unusual: a survey of 101 cases. Clin Exp Dermatol 2014;39(2):119-22

7. Brown CW Jr, Dy LC. Eccrine porocarcinoma. Dermatol Ther 2008 ;21(6):433-8

8. Edamitsu T, Minagawa A, Koga H, Uhara H, Okuyama R. Eccrine porocarcinoma shares dermoscopic characteristics with eccrine poroma: A report of three cases and review of the published work. J Dermatol 2016;43(3):332-5

9. Bombonato C, Piana S, Moscarella E, Lallas A, Argenziano G, Longo C. Pigmented eccrine poroma: dermoscopic and confocal features. Dermatol Pract Concept 2016 31;6(3):59-62 